\title{
An Interface between 3D Modeling Tool and Scientific Data Format
}

\author{
Md. Tarique Hasan Khan and Soonhung Han
}

\begin{abstract}
In these times scientific data format is used for versatile researches and wide varieties of computational work. Hierarchical Data Format (HDF) is such kind of powerful scientific data format, which can deal with very large dataset. HDF technologies address the problems of how to organize, store, discover, access, analyze, share and preserve data in the face of enormous growth in size and complexity. The aim of this research is to share as well as integrate the $3 \mathrm{D}$ modeling system's data with HDF. Computer programs supplied by the HDF group have been modified for the incorporation purpose with 3D modeling system. Result of this research indicates that, now it will be much easy to share the modeling data of $3 D$ tools with available other 3D modeling systems. Not only HDF but also other scientific data formats like CDF and NetCDF can be used in the near future for share and incorporation purpose with 3D modeling systems.
\end{abstract}

Index Terms-HDF, 3D modeling, scientific data format, hierarchy, incorporation, MATLAB.

\section{INTRODUCTION}

Scientific data format is of great use in the arena of data integration, storage, and interoperation as well as to manipulate various complex data. There are few scientific data formats nowadays which are widely used, like CDF (Common Data Format), FITS (Flexible Image Transport System), NetCDF (Network Common Data Format) etc. HDF (Hierarchical Data Format) is one of the widely used scientific data format.

HDF (Hierarchical Data Format) technologies are relevant when the data challenges being faced push the limits of what can be addressed by traditional database systems, XML documents, or in-house data formats. Leveraging the powerful HDF products and the expertise of the HDF Group, organizations realize substantial cost savings while solving challenges that seemed intractable using other data management technologies.

Many HDF $[2,4]$ adopters have very large datasets, very fast access requirements, or very complex datasets. Others turn to HDF because it allows them to easily share data across a wide variety of computational platforms using applications written in different programming languages. Some use HDF to take advantage of the many open-source and commercial tools that understand HDF.

An important arena of modeling and simulation [9] is 3D modeling. For varieties of design purpose 3D modeling tools

Manuscript received March 18, 2012, revised March 31, 2012.

Authors are with the Department of Ocean Systems Engineering, KAISIT (Korea Advanced Institute of Science and Technology), Korea (e-mail: tarique@kaist.ac.kr).
(ex-Google Sketch-up, 3D paint brush, 3D canvas and so on) are used. The main purpose of this research is to integrate the HDF with a 3D modeling tool because it deals with large and complex design data as well as it has to interoperate with different CAD systems. So to give the 3D modeling data more transportability or in other term it can be said that to make the data more preserved and interoperable, it is a must to integrate 3D modeling data with a Scientific Data Format like HDF.

\section{INTERIOR OF HDF TECHNOLOGY}

The Hierarchical Data Format (HDF) implements a model for managing and storing data and HDF technology comprises the following basic parts.

\section{A. Abstract Data Model}

It is a conceptual model of data, data types, and data organization. The abstract data model is independent of storage medium or programming environment.

\section{B. Storage Model}

It is a standard representation for the objects of the abstract data model. The HDF5 File Format Specification defines the storage model.

\section{Programming Model}

It is a model of the computing environment and includes platforms from small single systems to large multiprocessors and clusters. The programming model manipulates (instantiates, populates, and retrieves) objects from the abstract data model.

\section{Library}

It is the concrete implementation of the programming model. The Library exports the HDF5 APIs as its interface. In addition to implementing the objects of the abstract data model, the Library manages data transfers from one stored form to another. Data transfer examples include reading from disk to memory and writing from memory to disc.

\section{E. Stored Data}

It is the concrete implementation of the storage model. The storage model is mapped to several storage mechanisms including single disk files, multiple files (family of files), and memory representations.

\section{F. Application Program}

It uses data structures that represent the problem and algorithms including variables, tables, arrays, and meshes among other data structures. Depending on its design and function, an application may have quite a few different kinds of data structures and different numbers and sizes of objects. 


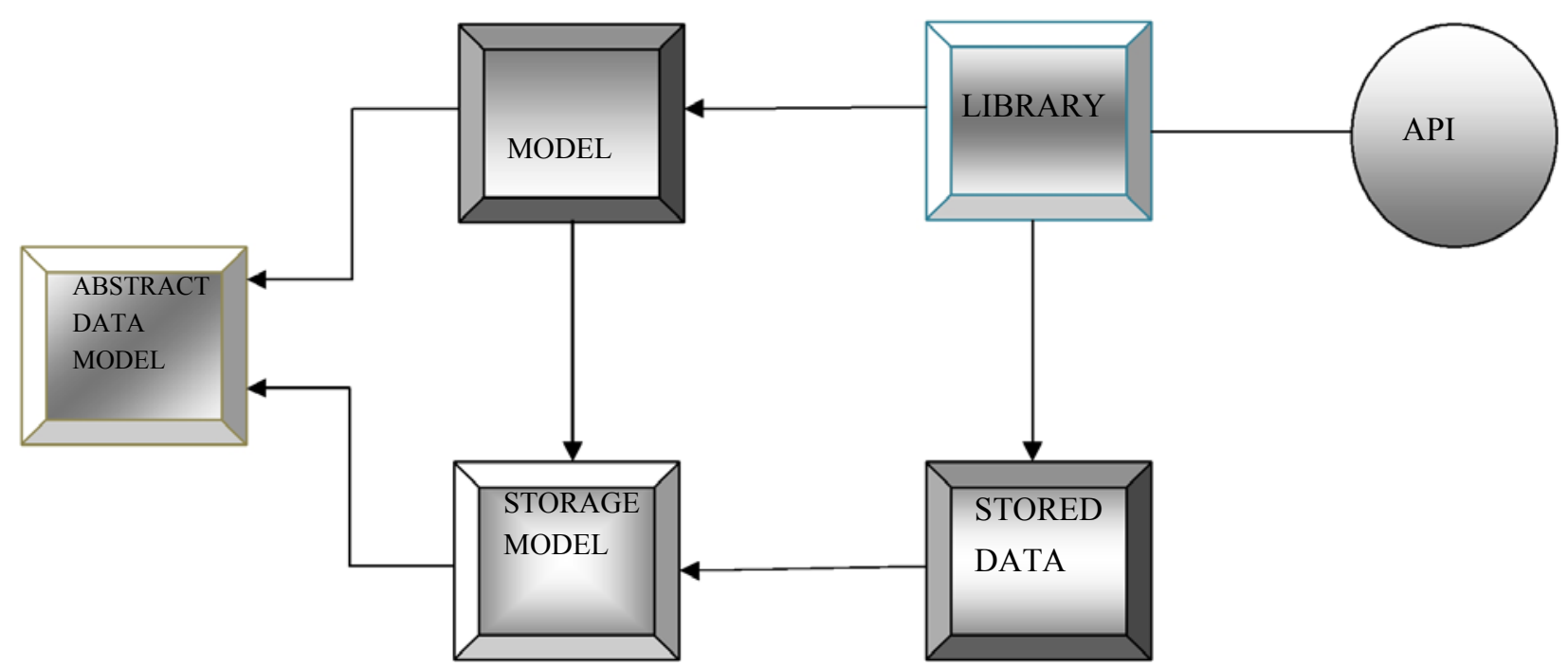

Fig. 1. HDF5 Technology in Detail $[6,11]$

\section{INTERIOR OF 3D MODELING TECHNOLOGY}

Before moving forward it should be mentioned that, the design procedure in the $3 \mathrm{D}$ modeling tool is being done by two methods usually. Firstly by using the built-in options provided by the tool itself and secondly by using the script file for that software system.

Here we are mostly concerned about the modeling by scripting because this technology has been manipulated to interoperate 3D modeling tools with the helpc of HDF. From Fig. 2 a brief idea can be found about modelincg using script language.

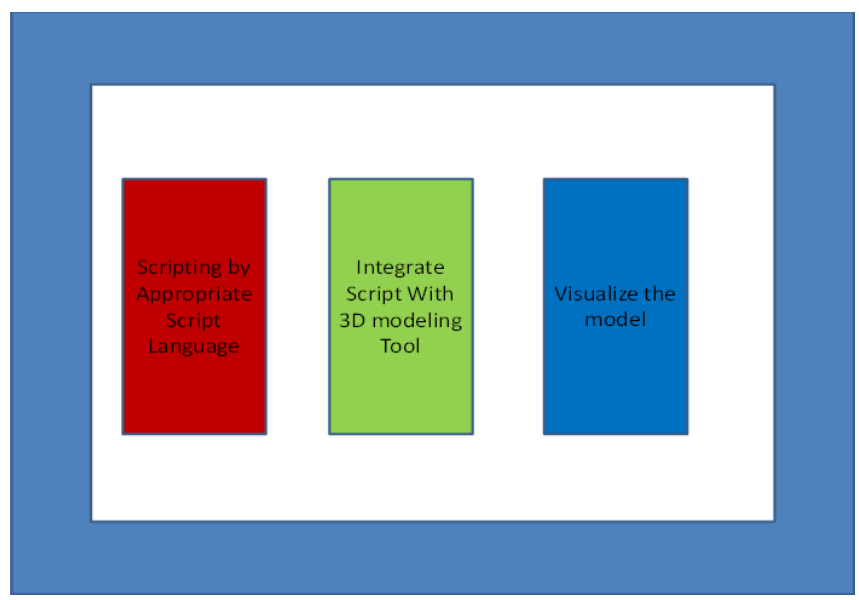

Fig. 2. Technology of modeling by scripting [10]

\section{A. Step 1}

In this stage appropriate scripting language will have to use for appropriate modeling tools, for instance Ruby scripting language is being used for Google Sketch-up. Similarly max-script is being used for 3D G-max.

\section{B. Step 2}

This step can be divided into two stages. In the first stage for the desired design required script will have to be written. Suppose for Google sketch-up Ruby language will have to be used properly so that, sketch-up can recognize the language.
In the second stage script language will have to be integrated with the modeling tool. For instance in Google sketch-up plug-in option is being used to recognize the Ruby script to the 3D modeling tool.

\section{Step 3}

This is the last step. In this step 3D tool will recognize the Ruby script and desired model will be viewed by the 3D-modeling-tool.

\section{PROBLEM DESCRIPTION}

For this research work Google sketch-up has been used as a 3D modeling tool to integrate it with HDF. For scripting purpose, that means to make model by scripting Ruby Script language has been used. In the Fig 3 model of stair and ground mesh is being seen and these models were built using Ruby script. In Fig 4 part of the Ruby script is being shown. Left scripting is for building the stairs and right one is to make the ground mesh. So, our research aim is to make a bridge between HDF and Google Sketch-up, so that information from the Sketch-up can be transported to HDF.

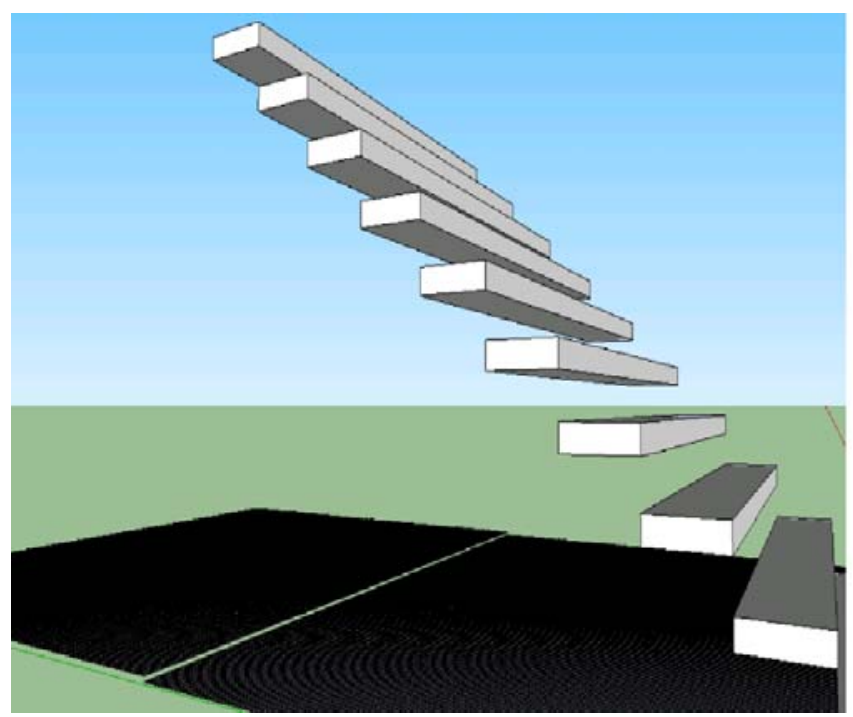




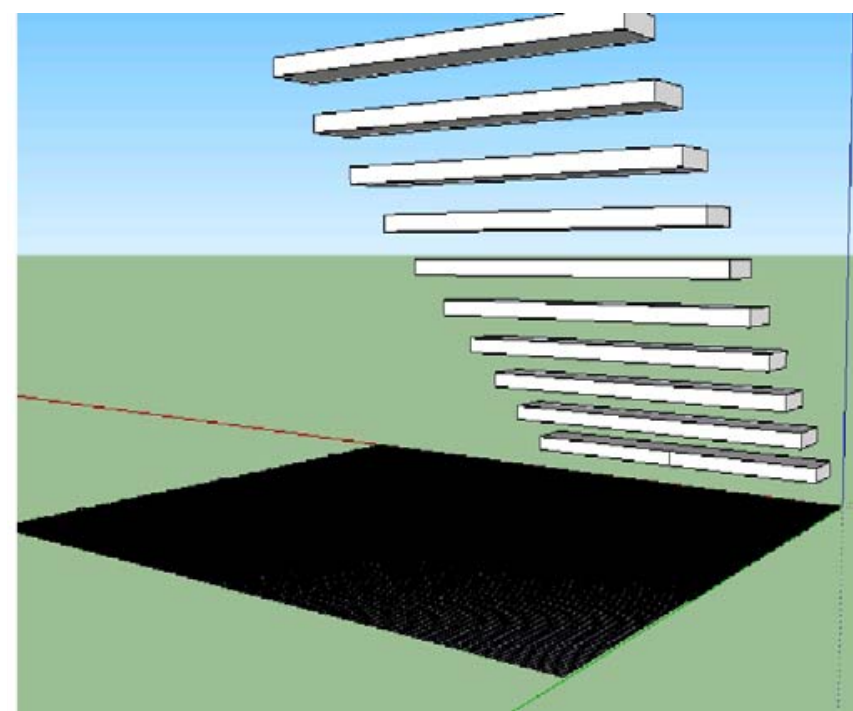

Fig. 3. Modeling using Ruby Script $[1,10]$

start_time $=$ Time.new
Sketchup.active_model.start_operation("Dra
w a grid", true)
for $x$ in $0 . .50$
for $y$ in $0 . .50$
p1 $=[\mathrm{x}, \mathrm{y}]$
p2 $=[\mathrm{x}+1, \mathrm{y}]$
$\mathrm{p} 3=[\mathrm{x}+1, \mathrm{y}+1]$
$\mathrm{p} 4=[\mathrm{x}, \mathrm{y}+1]$
def draw_stairs
\# Create some variables.
stairs $=10$
thise $=8$ run
collection it contains.
$=12$ width
thickess $=3$

Fig. 4. Part of Ruby Script for designing Stair (L) and Ground mesh (R) [1]

\section{REASON OF USING HDF}

There are many scientific data format available nowadays. Among all of them HDF is the most prominent scientific data format because of its speedy structure. There are other scientific data formats available nowadays but comparing with those formats HDF possesses much more positive points, for which it is regarded as the best one. A comparison table has been introduced in this section which compares HDF with other formats like CDF (Common data Format), NetCDF (Network Common Data Format), and FITS (Flexible Image Transportation System). The criterion with which HDF has been compared is as follows library function, programming effort, Image processing ability, Data intake ability and Hierarchical representation.

From the Table I first point of comparison is library function and in this criterion it is found out that for CDF, its library function is already obsolete that means less potent library function it is possessing. For NetCDF[5], it is found out that NetCDF has much potential library function than CDF. FITS has a rich library function but the most rich library function is possessed by the HDF. So in this sector HDF is the most prominent one. Second criterion for comparing is programming effort and in this section it is found it out that almost all of the scientific data format needs less programming effort to implement except the CDF.

Next criterion for comparison is image processing capability and in this sector it is found out that except CDF all the other can do good image processing. Next one is data intake ability and in this section also HDF possesses the most perfect ability to intake and process data perfectly. Last criterion is the hierarchical data representation and here only HDF can represent data in a hierarchical fashion.

\section{Solving Method \& ImPlementation}

To integrate between the two systems HDF group provides various codes which are written in MATLAB, C, FORTRAN and JAVA (partially). But MATLAB provides some built-in function like h5read, h5info, h5write, h5disp and so on. Due to these extra facilities provided by MATLAB (file extension .m) this system was chosen for the integration purpose. Sample name of code is given in Fig: 5 (L).

According to the Fig 5 (R) given above, for implementation, Ruby Script [13] of Google Sketch-up was first analyzed and necessary information's were extracted from that. Information from Ruby Script was read by a HDF generating code and then it was saved in the HDF file format, with an extension of .h5.HDF information were arranged in two different hierarchy, firstly non-dimensional information ( mesh data, line width, surface roughness etc.) and secondly, dimensional information (length, diameter, curvature, fillet etc. 


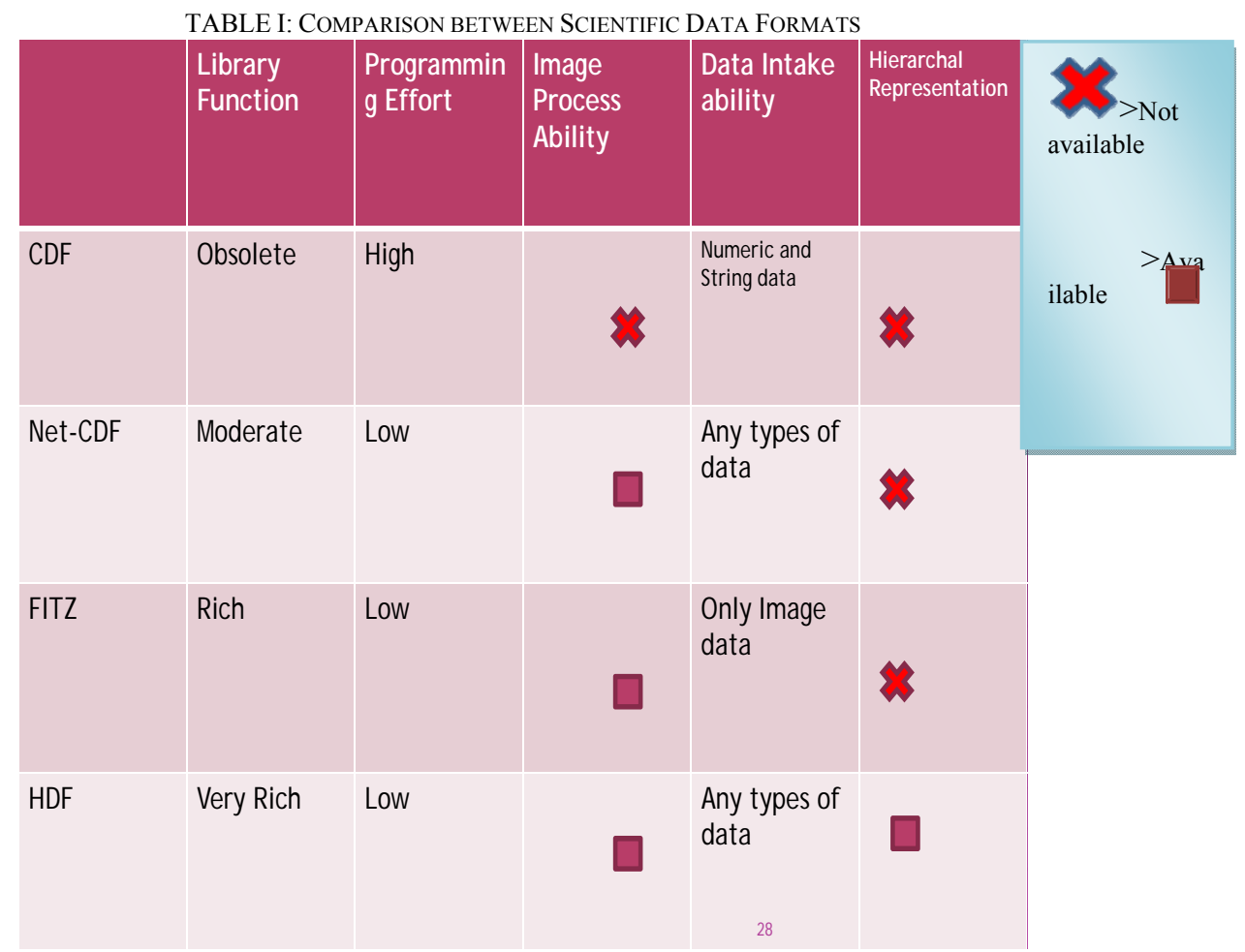

\section{Datasets:}

Set Space Allocation Time for Dataset:h5ex_d_alloc.m

\section{[ Output ] \\ [ HDF5 File ]}

Read / Write Dataset using Fletcher32 Checksum Filter: h5ex_d_checksum.m

[Output ] [ HDF5 File ]

Read / Write Chunked Dataset:

h5ex_d_chunk.m

[Output ] [ HDF5 File ]

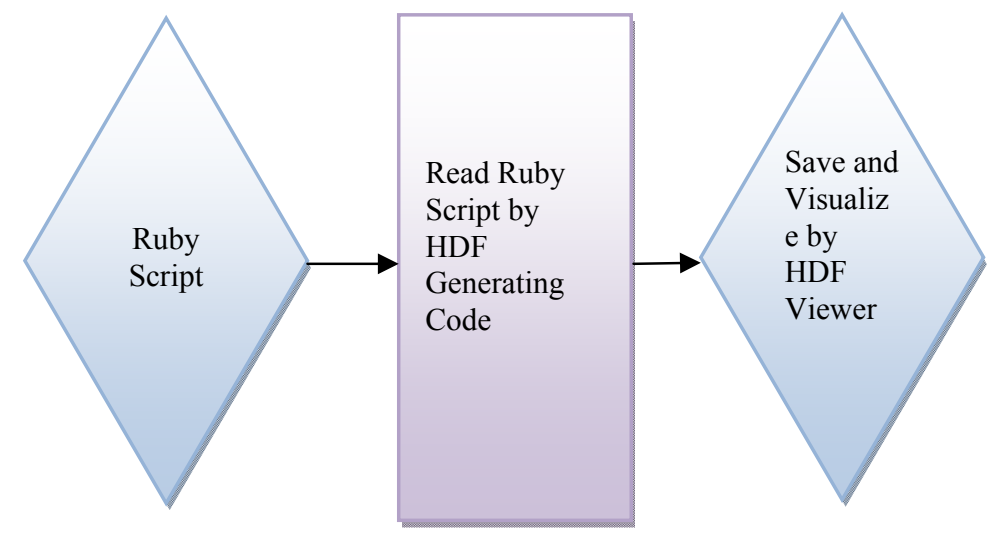

Fig. 5. Part of MATLAB code from HDF group (L) \& Implementation Pathway (R)[1]

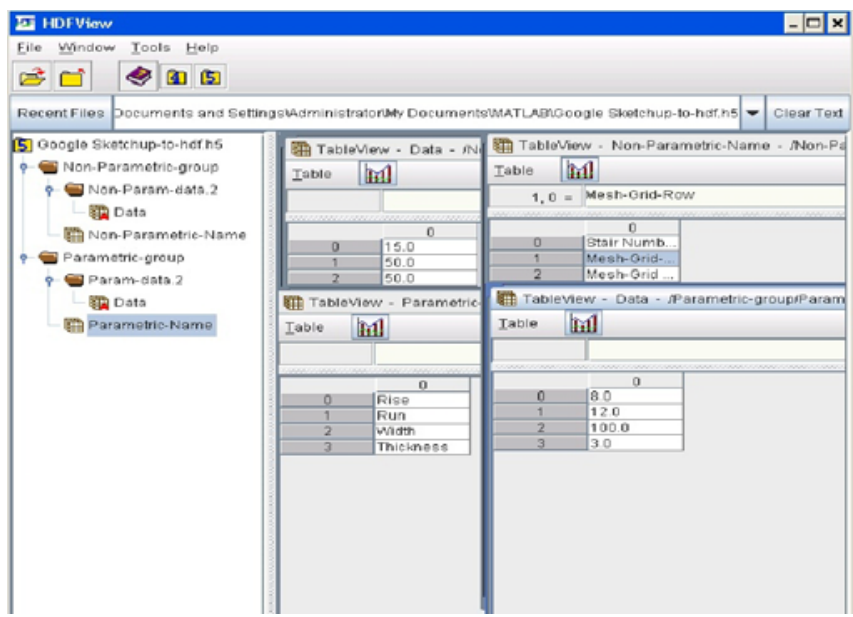

Fig. 6. Output view in the HDF viewer[1]

\section{AJOR CONTRIBUTION AND ADVANTAGE OF THIS RESEARCH}

Major contribution of this research is in the arena of interoperability. This work is just the initiator of a new method of interoperability between two 3D modeling [3, 7] systems using a well known scientific data format (HDF). In future HDF will act as a neutral format between two 3D modeling systems. In that system information from 3D modeling system-one will be sent to HDF (already done) and then it will be sent to the 3D modeling system-two. Interoperation will be done by this way.

\section{ACKNOWLEDGEMENT}

This work was supported by the Human Resources 
Development of the Korea Institute of Energy Technology Evaluation and Planning (KETEP) grant funded by the Korea government Ministry of Knowledge Economy. (No. 20114030200040) and also it was supported by WCU (World Class University) program through the National Research Foundation of Korea funded by the Ministry of Education, Science and Technology (R31-2008-000-10045-0) which was accomplished by iCAD laboratory of OSE (Ocean Systems Engineering); KAIST.

\section{REFERENCES}

[1] Khan, T. H. and Han S.; Incorporating 3D Modeling System with HDF (Hierarchical Data Format) for the Enhancement of Modeling, "4th International Conference on Computer Modeling and Simulation (ICCMS 2012), February 2012, Hong Kong” IPCSIT Vol.22 pp.169-173

[2] Folk, M., McGrath, R.E. and Yeager, N. HDF: An update and future direction "Geosciences and remote sensing symposium" Vol.1, No.1, pp.2733-275, 1999

[3] Yang, J. and Han, S. Repairing CAD model errors based on the design history "Computer-Aided Design" Vol. 38, No.6: pp.627-640, 2006

[4] Poinot, M. Five Good Reasons to Use the Hierarchical Data Format, "Computing in Science \& Engineering" Vol.12, No.5, pp. 84-90,2010

[5] Rew, R. and Davis, G. NetCDF: an interface for scientific data access. "Computer Graphics and Applications, IEEE" 1990. Vol.10 pp. 76-82

[6] Khan, T. H. and Han S. Integration of HDF (Hierarchical Data Format) and CAE, "CAD/CAM Conference Proceedings," Society of CAD/CAM Engineers of Korea, Jan. 2012.

[7] Yoo, B. and Han, S. Representation of Urban Buildings Using Modified Relief Mapping "Journal of Computer Science and Technology (SCI)" Vol.21, No.2: pp. 204-208, 2006

[8] Mun, D. ; Hwang, J ; Han, S. ; Seki, H. and Yang, J. Sharing product data of nuclear power plants across their lifecycles by utilizing a neutral model "Analysis of Nuclear Energy(SCI)" Vol.35, No.2, pp.175-186, 2008.
[9] Ciepiela, E.; Harężlak, D.; Kocot, J.; Bartyński, T.; Kasztelnik, M.; Nowakowski, P. Exploratory programming in the virtual laboratory "IMCSIT Proceeding" pp. 621-628, 2010

[10] Khan, T. H. and Han S. An Approach of Interoperability between 3D Modeling Systems Using the Scientific Data Format HDF "The Visual Computing Journal", 2012 (will be submitted)

[11] Lee, K. and Spence, P.L. View HDF: visualization and analysis tool for hierarchical data format files "OCEANS '02 MTS/IEEE" Page(s): 744 - 750 vol.2, 2002

[12] Yang, W. and Di, L. Georectification of HDF-EOS swath data "Geoscience and Remote Sensing Symposium, 2002. IGARSS '02. 2002 IEEE International” pp. 1349 - 1351 vol.3, 2002

[13] He, C.; Jianguo, G. and Ping, P. Experiment and Research of Google SketchUp Combine with ArcGIS in the Three-Dimensional Urban Geographic Information System "Software Engineering, 2009. WCSE '09. WRI World Congress on" pp. 309-312 vol.2, 2009

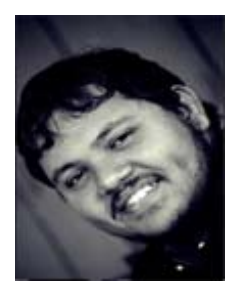

Md. Tarique Hasan Khan obtained a first class B.Sc. (Hons) degree in Mechanical Engineering from the BUET (Bangladesh University of Engineering and Technology), Bangladesh in 2009. He is currently a MSc. candidate student at KAIST (Korea Advanced Institute of Science and Technology) from the department of OSE (Ocean Systems Engineering); His current research interests include CAD, Scientific Data Format and CAE.

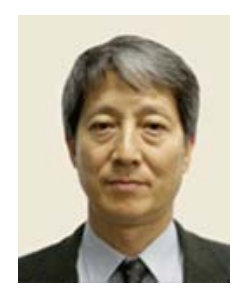

Soonhung Han is the Chair of the Department of Ocean Systems Engineering and a Professor in the Department of Mechanical Engineering of KAIST. $\mathrm{He}$ is leading the Intelligent CAD Laboratory of KAIST and the STEP Community of Korea. His research interests include ISO standard for the exchange of product model data (STEP), virtual reality (VR) for engineering design and knowledge-based design systems. His domain of interests include automotive and shipbuilding. 